\title{
Observing sub-Poissonian statistics of twisted single photons using oscilloscope
}

Cite as: Rev. Sci. Instrum. 90, 113104 (2019); https://doi.org/10.1063/1.5109544

Submitted: 09 May 2019 . Accepted: 03 November 2019 . Published Online: 21 November 2019

Nijil Lal (iD, Biveen Shajilal, Ali Anwar (D), Chithrabhanu Perumangatt (iD), and R. P. Singh
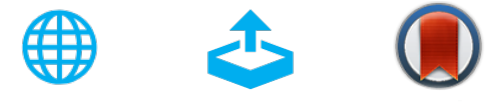

\section{ARTICLES YOU MAY BE INTERESTED IN}

Space- and time-resolved UV-to-NIR surface spectroscopy and 2D nanoscopy at $1 \mathrm{MHz}$ repetition rate

Review of Scientific Instruments 90, 113103 (2019); https://doi.org/10.1063/1.5115322

Single-pass non-destructive electronic detection of charged particles

Review of Scientific Instruments 90, 113301 (2019); https://doi.org/10.1063/1.5110988

A simple method for in situ measurement of vacuum window birefringence

Review of Scientific Instruments 90, 113001 (2019); https://doi.org/10.1063/1.5121568

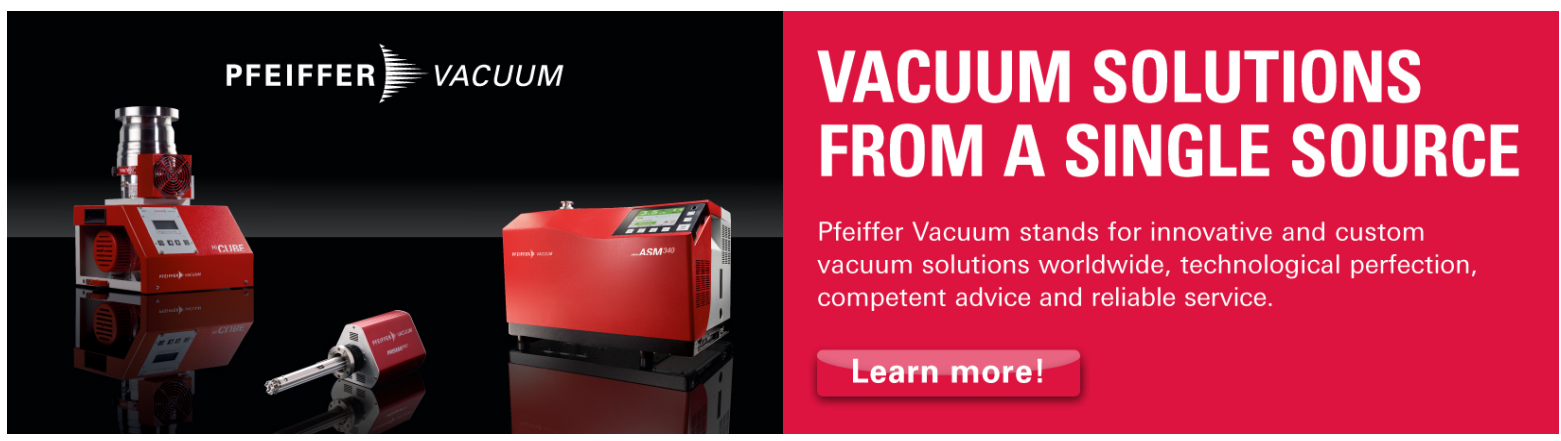




\title{
Observing sub-Poissonian statistics of twisted single photons using oscilloscope
}

\author{
Cite as: Rev. Sci. Instrum. 90, 113104 (2019); doi: 10.1063/1.5109544 \\ Submitted: 9 May 2019 • Accepted: 3 November 2019 • \\ Published Online: 21 November 2019
}

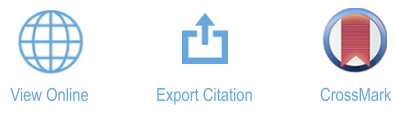

Nijil Lal, $^{1,2, a)}$ (D) Biveen Shajilal, ${ }^{3, b)}$ Ali Anwar, ${ }^{1, c)}$ (D) Chithrabhanu Perumangatt, ${ }^{1, c)}$ (D) and R. P. Singh ${ }^{1}$

AFFILIATIONS
${ }^{1}$ Physical Research Laboratory, Ahmedabad 380009, India
${ }^{2}$ Indian Institute of Technology, Gandhinagar 382355, India
${ }^{3}$ Cochin University of Science and Technology, Kochi 682022, India

a) Electronic mail: nijil@prl.res.in

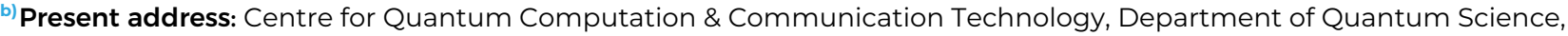

The Australian National University, Canberra, ACT 2601, Australia.

${ }^{c}$ Present address: Centre for Quantum Technologies, National University of Singapore, 3 Science Drive 2, 117543, Singapore.

\begin{abstract}
Heralded single photon sources (HSPSs) from spontaneous parametric down-conversion are widely used as single photon sources. We study the photon number statistics of an HSPS carrying orbital angular momentum in our laboratory and observe the sub-Poissonian statistics using only photodetectors and an oscilloscope.
\end{abstract}

Published under license by AIP Publishing. https://doi.org/10.1063/1.5109544

\section{INTRODUCTION}

The photon is a prominent qubit candidate in quantum information processing (QIP). Most of these applications require a single photon source (SPS) which will provide an "on-demand," deterministic supply of photons. Such ideal SPSs can be realized in crystal color centers, ${ }^{1}$ quantum dots, ${ }^{2}$ single atoms, ${ }^{3}$ single ions, ${ }^{4}$ and single molecules. ${ }^{5}$ The photon being an indivisible quantum of energy and the consequent impossibility of copying associated information from an unknown arbitrary state makes single photon sources an ideal choice for quantum information processing, secure communication, and metrology. Spontaneous parametric down-conversion (SPDC) is one of the most widely used processes to generate single photon sources as it provides a robust and bright source at room temperature. ${ }^{6}$ In this process, a pump photon is down-converted in frequency in a $\chi^{(2)}$ nonlinear crystal to a pair of photons, conventionally called the signal $(s)$ and the idler $(i)$. The detection of one photon from the pair heralds the presence of another, and this conditioned detection serves as a technique for generating single photon sources. SPDC is also important in QIP as a bright source of entangled photon pairs and thus finds applications in quantum teleportation, quantum cryptography, ${ }^{9}$ and quantum dense coding. ${ }^{10}$
A single photon source shows nonclassical properties such as sub-Poissonian photon number distribution and antibunching. The inherent thermal nature of the photon distribution in the individual arms (i.e., signal or idler) evokes the possibility of occurrences of multiple photons and hence would qualify the source as less quantum than it is expected to be. The occurrence of multiple photon pairs and consequent photon bunching threatens the security in cryptography related applications. Thus, it is important to study and quantify the nonclassicality of the single photon sources being used. A general classification puts the sources into three categories, super-Poissonian, Poissonian, and sub-Poissonian, according to the photon number distribution. Classical sources, such as thermal and coherent radiation, show super-Poissonian and Poissonian statistics, respectively, while certain nonclassical sources, such as a single photon source, show sub-Poissonian distribution. Violations of bounds defined for the number statistics of classical sources imply the nonclassical behavior of single photon sources. ${ }^{11-14}$ Photon antibunching is another manifestation of the nonclassical behavior of the source and can be observed through the measurement of the degree of second-order coherence, $g^{(2)}(\tau)$. Single photon sources show antibunching, and hence, $g^{(2)}(0)$ falls to zero. Although antibunching and sub-Poissonian statistics are completely nonclassical 
effects and tend to occur together in many systems, they are distinct effects and need not necessarily be associated with one another. ${ }^{15,16}$ For a heralded SPS, antibunching can be observed using a heralded Hanbury Brown-Twiss (HBT) type experiment. ${ }^{17,18}$ Twisted single photons carrying orbital angular momentum (OAM) are gaining interest in quantum information processing as they provide infinite number of orthogonal states to encode information. ${ }^{19,20}$ The statistical second order correlation of classical beams ${ }^{21,22}$ as well as photons $^{23}$ carrying OAM has already been studied. In the present work, we verify the sub-Poissonian nature of heralded single photons generated in parametric down-conversion having Gaussian as well as higher order OAM using a simple setup consisting of photodetectors and an oscilloscope.

\section{THEORY}

One can define an ideal single photon source as a Fock state for which the number statistics gives mean, $\mu=1$, and variance, $\sigma^{2}=0$. Treatment of the electromagnetic field as a quantum harmonic oscillator allows us to define quadrature field operators, $\hat{X}_{1}$ and $\hat{X}_{2}$, which can be shown to follow the uncertainty relation, ${ }^{24}\left\langle\Delta \hat{X}_{1}^{2}\right\rangle\left\langle\Delta \hat{X}_{2}^{2}\right\rangle$ $\geq 1 / 16$. The minimum uncertainty state, with $\left\langle\Delta \hat{X}_{1}^{2}\right\rangle=\left\langle\Delta \hat{X}_{2}^{2}\right\rangle$, is the coherent state,

$$
|\alpha\rangle=\exp ^{\frac{-|\alpha|^{2}}{2}} \sum_{n=0}^{\infty} \frac{\alpha^{n}}{\sqrt{n !}}|n\rangle,
$$

which defines a Poissonian distribution of photon number, ${ }^{25} n$, with $\left\langle(\Delta \hat{n})^{2}\right\rangle=\langle\hat{n}\rangle$. Squeezing these states in terms of number or phase is achieved when the respective variance is less than that corresponding to the coherent state. The photon number variance can be written as

$$
\left\langle\Delta \hat{n}^{2}\right\rangle=\langle\hat{n}\rangle+\left\langle\hat{a}^{\dagger} \hat{a}^{\dagger} \hat{a} \hat{a}-\left\langle\hat{a}^{\dagger} \hat{a}\right\rangle^{2}\right\rangle
$$

where $\hat{a}$ and $\hat{a}^{\dagger}$ are the annihilation and creation operators. Based on the variance, the Mandel Q-parameter ${ }^{26}$ is defined by

$$
Q \equiv \frac{\left\langle\hat{a}^{\dagger} \hat{a}^{\dagger} \hat{a} \hat{a}-\left\langle\hat{a}^{\dagger} \hat{a}\right\rangle^{2}\right\rangle}{\left\langle\hat{a}^{\dagger} \hat{a}\right\rangle}=\frac{\left\langle(\Delta \hat{n})^{2}\right\rangle-\langle\hat{n}\rangle}{\langle\hat{n}\rangle} .
$$

It classifies light sources on the basis of photon number fluctuations. $Q \geq 0$ for coherent and all other classical sources of light, while sub-Poissonian light is identified with $Q<0 .^{26,27}$
The detected photoelectron statistics and the statistics of the incident photons can be different depending upon various factors such as the coherence time of the incident light, detector dead-time, and efficiency of detection. The first two are taken care of while setting the binning window and pump intensity, respectively. This is discussed in detail in Sec. III. Correcting for the efficiency, the mean and standard deviation for the detected-photon distribution can be written as ${ }^{28}$

$$
\begin{gathered}
\langle m\rangle=\eta\langle n\rangle, \\
\sigma^{2}(m)=\eta^{2} \sigma^{2}(n)+\eta(1-\eta)\langle n\rangle,
\end{gathered}
$$

where $m$ is the detected photocounts, $n$ is the actual photocounts, $\eta$ is the detector quantum efficiency, and $\sigma^{2}$ is the variance. Hence, the expression for the Fano factor will be

$$
F_{n}=\left(F_{m}-(1-\eta)\right) / \eta \text {. }
$$

Correcting for the efficiency of detection, the Q-parameter will be

$$
Q_{n}=Q_{m} / \eta \text {. }
$$

\section{EXPERIMENT}

The experimental setup used for building the statistics of the heralded single photon source is given in Fig. $1 .^{29}$ A $405 \mathrm{~nm}$ diode laser (Toptica TopMode) is used as a pump to generate photon pairs from a Type-I BiBO crystal (Castech, $5 \mathrm{~mm}$ thickness). The polarization of the pump is oriented along the crystal axis using a half-wave plate. The output pairs are spectrally filtered using interference filters of passband of $810 \pm 5 \mathrm{~nm}$ to rule out the nondegenerate pairs. Two diametrically opposite regions are selected from the cone of correlated pairs of photons generated in the Type-I SPDC. These individual arms (signal and idler) are then collimated and coupled to a single mode fiber (SMF, idler) and a multimode fiber (MMF, signal) through a fiber coupler (CFC-5X-B).

The individual signal and idler photons are detected using single photon detectors. We have used two single photon counting modules (SPCMs, Excelitas SPCM-AQRH-16-FC) placed in both signal and idler arms for the detection of the photon pairs. Photomultiplier tubes (PMTs), which will be more common in laboratories than an SPCM can also be used for the detection. The coincidences are maximized by optimizing the coupling to the fiber. The

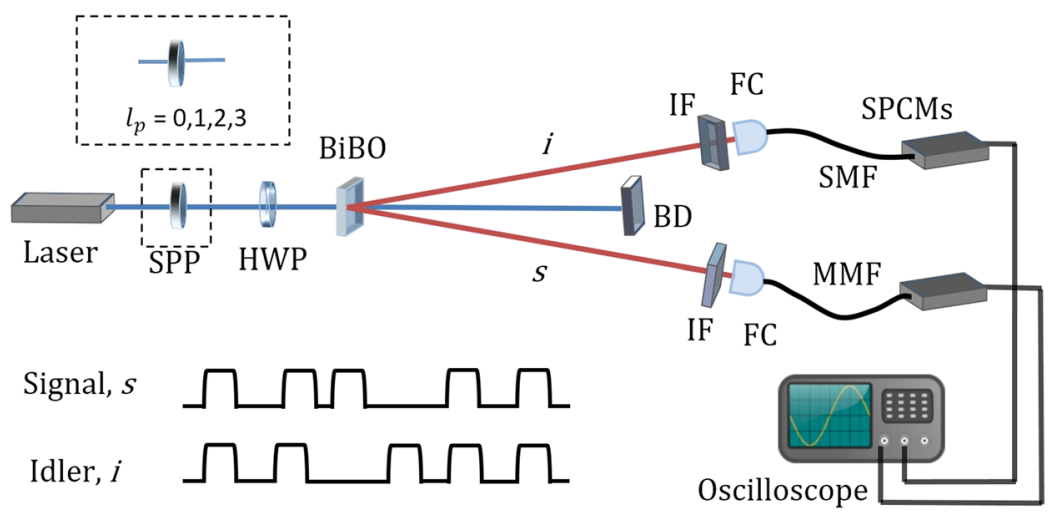

FIG. 1. Experimental setup to determine the photon number statistics of the heralded PDC source. HWP-half wave plate, BiBO-nonlinear crystal, BD-beam dump, IF-interference filters, FC-fiber couplers, and SPCMssingle photon counting modules. Different OAM orders can be imparted to the heralded single photon by placing SPPs of different orders in pump and projecting the idler photon in a single mode fiber. 
SPCM (or PMT) generates a TTL pulse in response to each photon incidence. However, the total number of photon incidences that can be recorded by a detector is limited by the detector dead time. If more than two photon incidences occur at the detector within the dead-time, the detector will not be able to register the second detection and the number statistics will not give the correct distribution. ${ }^{30,31}$ Therefore, it is important to verify that the probability of such multiple detection within the dead-time is negligible. The individual count rates are $\sim 10^{4} / \mathrm{s}$ for the single mode fiber and $\sim 10^{5} / \mathrm{s}$ for the multimode fiber (MMF). The detector dead time is $27 \mathrm{~ns}\left(t_{d}\right)$ for the SPCMs used in the setup. If $n$ is the total count rate in the detector associated with MMF (being larger in value compared to the SMF) and $t_{d}$ is the dead time corresponding to the detector, then $n \ll 1 / t_{d}$ ensures that the probability that more than two photons arriving within the dead time interval is very less. Our count rates are such that this probability is negligible and dead time effects on the total counts can be ignored. In addition to this, since the detectors being used are not photon number-resolving, the output pulses can correspond to the incidence of one or more photons. The pump power is kept in the low power regime $(1 \mathrm{~mW})$ in order to avoid such scenarios due to the possible multiple photon generations. ${ }^{32}$

The output waveform from each arm of the SPDC is recorded using a digital oscilloscope (Infiniium 90000A series) for an exposure time of $20.5 \mathrm{~ms}$. Ten iterations of such time series have been recorded to calculate the $Q$-parameter. Each iteration records a time series of $20.5 \mathrm{Mpts}$ and a resolution of $1 \mathrm{~ns}$. Oscilloscopes with lower specifications can also be used but with compromising the length of the time series to be captured and time resolution. In order to build the statistics, the detection events at the photodiodes are counted within a defined time interval (binning window) and the distribution of the number of such occurrences is obtained. This is done by converting the analog output from the oscilloscope into a series of 1's and 0 's which corresponds to the presence or absence of a detection, respectively. We built a MATLAB code which identifies an event pulse in the oscilloscope output time series and labels its onset as 1 and the rest of the series as 0 . This generates a binary time series of events in terms of the occurrences or nonoccurrences of photon detection (Fig. 2). A probability distribution of events is built from this generated time series.

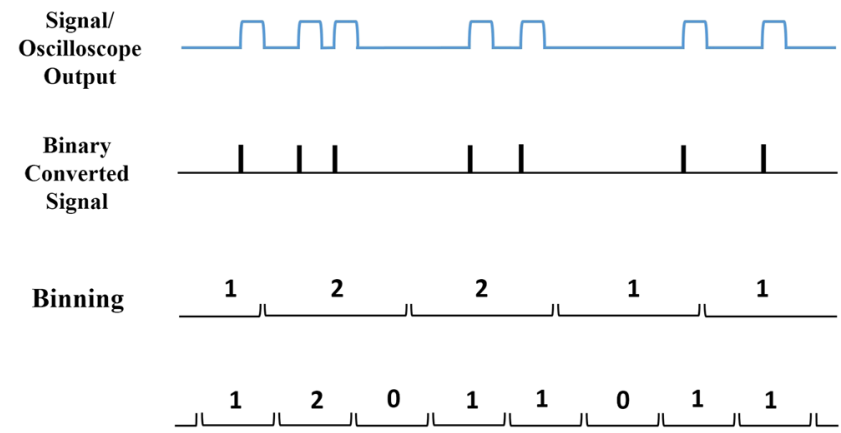

FIG. 2. Building the number statistics from the recorded time series. The oscilloscope output is converted into binary time series of 0 's and 1's that represent the presence (1) or absence (0) of a detected photon. Corresponding probability distributions are built from this series by defining an appropriate binning window size.
The time series of binaries generated for both the signal and idler are then sliced into smaller binning windows in such a way that statistically one detection falls in a bin. The number distribution is developed for these individual arms. A new time series corresponding to the coincidences of photon pairs is developed from the time series corresponding to the signal and idler, in a way that a count is observed in one arm (within the coincidence window, $\tau_{c}=10 \mathrm{~ns}$ ), given a detection in the other arm. The number distribution of this new time series corresponds to the statistics of the heralded single photon source from SPDC. Another important factor to be taken care of is the coherence time of the input pump field. If the binning window is considerably larger than the coherence time, the statistics of the photoelectrons will result in a Poissonian distribution independent of the inherent photon statistics. ${ }^{33}$ Since we are taking two photon coincidence here, the coherence of the pump will determine the coherence of paired down-converted photons. The pump coherence time is $80 \mathrm{~ns}$, which in turn results in a coherence time of $\sim 80 \mathrm{~ns}$ for the biphoton output of the SPDC. The binning window (20 ns) defined for building the coincidence series during postprocessing is much within this two photon coherence time. The MATLAB code to build the statistics from the recorded time series is shared in the GitHub repository for open access. ${ }^{34}$

Twisted single photons of different orders are obtained in SPDC by pumping with optical vortices of different orders and projecting the idler photon in a single mode fiber and hence selecting only those with OAM, $l=0$. Due to OAM conservation in SPDC, this will result in only those signal photons which have the same OAM as the pump contributing to the coincidence counts. ${ }^{35}$ Hence, a photon number distribution obtained for the coincidence events will correspond to the number statistics of single photons carrying OAM.

\section{RESULTS AND DISCUSSION}

Initially, to see the photon number distribution for a classical coherent source, we select a coherent laser source (Thorlabs $2 \mathrm{~mW}$ $\mathrm{HeNe}, 632.8 \mathrm{~nm}$ ). The intensity is attenuated such that the detected photocounts are below the saturation level of the detectors. The time series of events recorded using an oscilloscope was converted into a binary series of photon incidence events. A counting histogram that gives the probability of detection of $n$ events within the corresponding time bin is generated for this time series that shows Poissonian distribution (Fig. 3). For a time bin width such that an average number of less than one photon falls within the bin $(\mu=0.1)$, the variance $\left(\sigma^{2}\right)$ is determined to be 0.103 . The $Q$-parameter is calculated to be $0.045 \pm 0.03$ [for net efficiency, $\eta=0.66$ in Eq. (7)] which is close to a Poissonian distribution of photon numbers.

For the heralded single photon source, the number statistics is built from the binary series of coincidences generated by postprocessing the recorded events of individual signal and idler photons (Fig. 4). For $\mu=0.1$, the variance $\left(\sigma^{2}\right)$ for this source is calculated to be 0.0901 . The Mandel Q-parameter comes out to be -0.099 . The negative value of $Q$ is an indication of the sub-Poissonian behavior of the source. The detectors that are used in the experiments have a quantum efficiency of 0.6 at the spectral region in which the experiment has been undertaken. Along with this, taking the coupling efficiency of the fiber and other losses into account, the net efficiency 


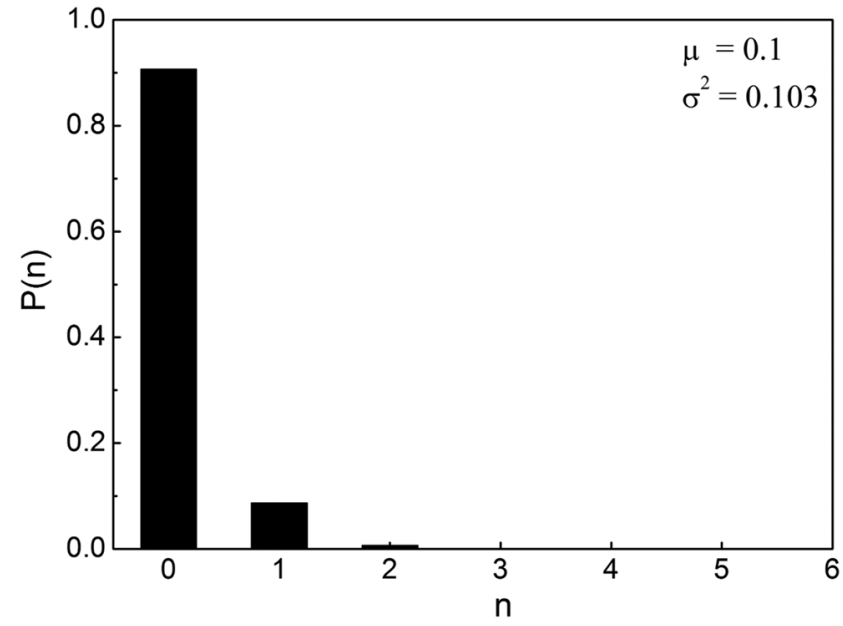

FIG. 3. Detected photon number distribution for a coherent source. Here, $n$ corresponds to the number of detected photons within a binning window, and $P(n)$ is the probability of obtaining $n$ detection events per bin. The mean photon number is approximately equal to the variance.

of detection is determined to be $\eta=0.3$. Incorporating this value, the $Q$-parameter for the heralded photon source is determined as -0.33 for the heralded single photons. A set of repeated measurements reveals the average value, $Q=-0.331 \pm 0.004$, for a heralded SPS generated using a Gaussian pump profile. $Q=-1$ implies an ideal single photon source where photons come out in order with a variance that equals to zero in their temporal distribution. Intuitively, $Q$ is expected to be less negative (i.e., reduced nonclassicality) for the case of heralded SPS as the pair generation is a spontaneous process and it would result in a nonzero variance. In addition to this, various losses randomize the detection of a HSPS and hence result in a

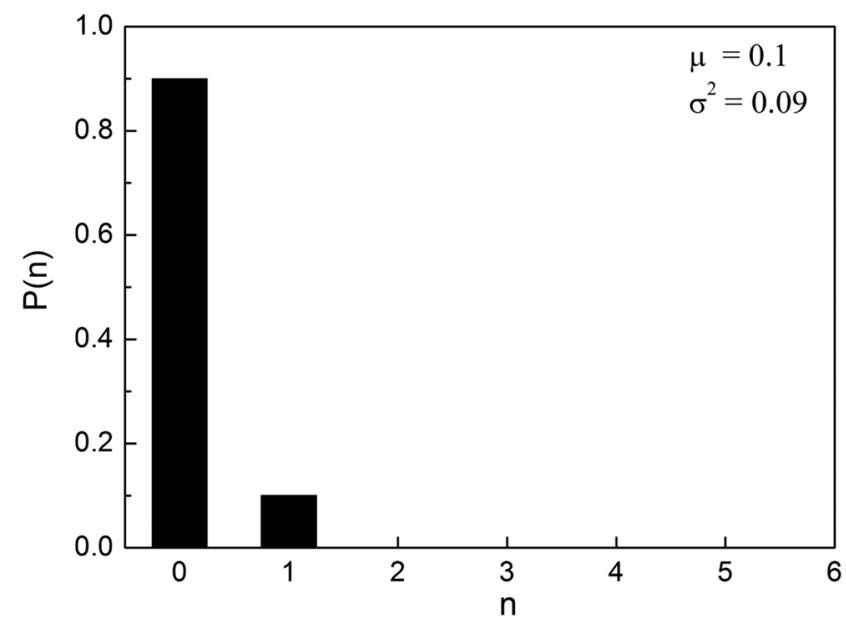

FIG. 4. Detected photon number distribution for a heralded single photon source. Here, $n$ corresponds to the number of coincidence detection within a binning window, and $\mathrm{P}(\mathrm{n})$ is the probability of obtaining $\mathrm{n}$ coincidence events per bin. reduced nonclassicality in measurement. While the intention of this work is not to formulate a rigorous theoretical model for the number statistics of heralded photons, we matched the experimentally observed data with the statistics of a simulated coincidence series. A coincidence series is generated from two correlated time series consisting only 0 and 1 which correspond to the individual idler and signal series. The correlated time series can be generated by randomly distributing 1's in a time series (such that the sum of 1's is matched with the experimentally observed single counts) and duplicating it. The random distribution of 1's replicates the spontaneous nature of the pair generation process. However, the actual scenario would differ from this as losses in the system would individually randomize these series further. After incorporating a loss of $\eta=0.3$ in each time series separately by randomly erasing $30 \%$ of 1 's, we build the coincidence series the same way we did during postprocessing of the experimental data 2. For parameters such as efficiency, binning window, coincidence window, total counts, and time series length
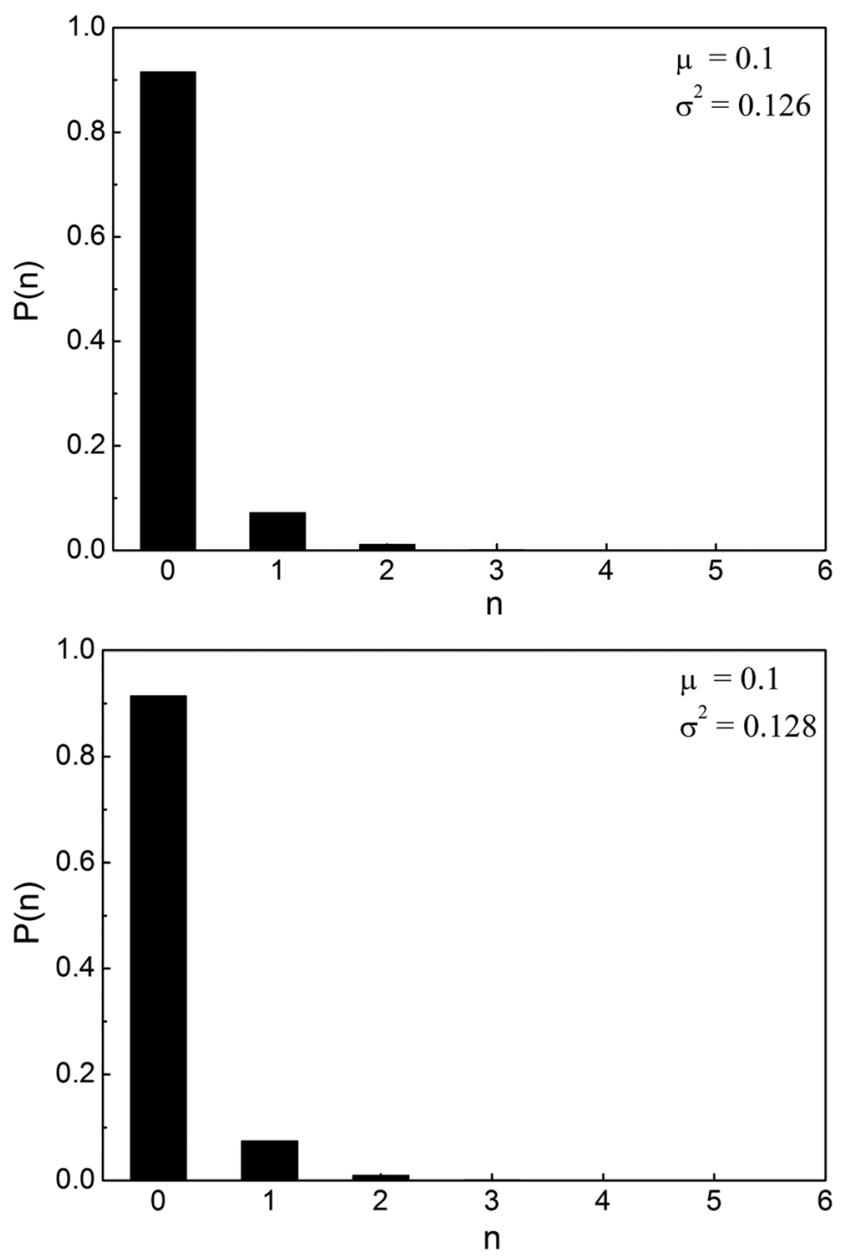

FIG. 5. Detected photon number distribution for thermal photons in the individual idler (top) and signal (bottom) arms of the downconverted pairs. Here, $\mathrm{n}$ corresponds to the number of single arm (idler/signal) photons within a binning window, and $P(n)$ is the probability of obtaining $n$ detection events per bin. 
matched with the corresponding experimentally observed values, the $Q$-parameter turns out to be -0.3 . Hence, the experimentally observed value of $Q$ for HSPS is reasonable with the predictions from a lossy model for spontaneously generated correlated pairs of photons.

In the same way, photon number histograms are generated for the individual arms of the down-converted pairs of photons (Fig. 5). For an average photon number $\mu=0.1$, the variances were 0.126 and 0.128 , respectively, for the signal and idler arms which are significantly apart from that corresponds to a Poissonian distribution. With $\eta=0.3$, the $Q$-parameter is calculated to be 0.87 and 0.93 for the idler and signal arms, respectively, with statistical errors of the order of $10^{-3}$. This super-Poissonian behavior is expected since the individual signal and idler photons from the down-conversion process are in a thermal state. ${ }^{33,36,37}$ Being thermal in nature, the single arm statistics is expected to be such that $Q=1$.

The Q-parameter for the heralded single photons having different OAM, obtained by pumping with an optical vortex and projecting the idler arm to $l=0$, are given in Table $\mathrm{I}$. The time series of photon detection events of $20.5 \mathrm{~ms}$ length are recorded using the oscilloscope. 10 such iterations are recorded to determine the Q-parameter within statistical errors. For heralded single photons, it is found to violate the classical bounds of number statistics (i.e., $Q \geq 0$ ) by $\sim 10$ standard deviations for photons carrying different OAM. The $Q$-parameter is observed to decrease with the increasing order of twisted photon OAM. This can be attributed to the reduction in the number of coincidence events with higher order pump as the singles in the idler arm (with $l=0$ ) decrease with pump order. The effects on the probability amplitude of the two photon coincidences and spectral bandwidth when pumped with different Laguerre-Gaussian modes is already discussed in the literature. $^{38,39}$

The background counts due to ambient stray light $(<100 \mathrm{cps})$ has been measured and subtracted from the detected counts. Afterpulsing probability for the detectors that are used is about $0.5 \%$ and is negligible. Ringing is taken care of by appropriately terminating the output pulse into a $50 \Omega$ load at the oscilloscope. Triggering from noise signals is also taken care of during postprocessing. This is done by keeping a threshold of $1.0 \mathrm{~V}$ while doing the conversion of the time series to binary series since the output TTL levels after BNC couplers are $>1.5 \mathrm{~V}$.

TABLE I. Mandel Q-parameter obtained for the photon number distributions of twisted single photons having different orders generated in parametric down conversion.

\begin{tabular}{cc}
\hline \hline Twisted photon OAM, $l$ & Q-parameter \\
\hline \multirow{2}{*}{0} & -0.331 \\
& \pm 0.002 \\
\multirow{2}{*}{1} & -0.336 \\
& \pm 0.003 \\
\multirow{2}{*}{2} & -0.343 \\
& \pm 0.005 \\
\multirow{2}{*}{3} & -0.353 \\
& \pm 0.008 \\
\hline \hline
\end{tabular}

\section{CONCLUSIONS}

Conventional quantum optics experiments to determine the nonclassical statistical behavior of single photon sources involve time-to-digital converters along with associated postprocessing. Here, we introduce a simple technique to build the number statistics of a heralded single photon source using a digital oscilloscope. The signal and idler in the SPDC output are detected and registered in the oscilloscope. From the recorded data, a time series corresponding to the coincident detection and the corresponding number distribution are obtained. In this way, one can observe the sub-Poissonian behavior of the photon number statistics of the source using a set of detectors and an oscilloscope available in almost all the optics laboratories. We also show that in this way we can determine the number statistics of heralded single photons carrying OAM and thus can characterize them for further applications involving OAM of single photons.

\section{REFERENCES}

${ }^{1}$ T. Gaebel, I. Popa, A. Gruber, M. Domhan, F. Jelezko, and J. Wrachtrup, “Stable single-photon source in the near infrared," New J. Phys. 6, 98 (2004).

2 A. J. Shields, "Semiconductor quantum light sources," Nat. Photonics 1, 215-223 (2007).

${ }^{3}$ M. Hennrich, T. Legero, A. Kuhn, and G. Rempe, "Photon statistics of a nonstationary periodically driven single-photon source,” New J. Phys. 6, 86 (2004).

${ }^{4}$ C. Maurer, C. Becher, C. Russo, J. Eschner, and R. Blatt, “A single-photon source based on a single Ca+ ion," New J. Phys. 6, 94 (2004).

${ }^{\mathbf{5}}$ M. Steiner, A. Hartschuh, R. Korlacki, and A. J. Meixner, "Highly efficient, tunable single photon source based on single molecules," Appl. Phys. Lett. 90, 183122 (2007).

${ }^{6}$ S. Fasel, O. Alibart, S. Tanzilli, P. Baldi, A. Beveratos, N. Gisin, and H. Zbinden, "High-quality asynchronous heralded single-photon source at telecom wavelength," New J. Phys. 6, 163 (2004).

${ }^{7}$ M. V. Jabir and G. K. Samanta, "Robust, high brightness, degenerate entangled photon source at room temperature," Sci. Rep. 7, 12613 (2017).

${ }^{8}$ D. Boschi, S. Branca, F. De Martini, L. Hardy, and S. Popescu, "Experimental realization of teleporting an unknown pure quantum state via dual classical and Einstein-Podolsky-Rosen channels,” Phys. Rev. Lett. 80, 1121 (1998).

${ }^{9}$ N. Gisin, G. Ribordy, W. Tittel, and H. Zbinden, “Quantum cryptography," Rev. Mod. Phys. 74, 145 (2002).

${ }^{10}$ K. Mattle, H. Weinfurter, P. G. Kwiat, and A. Zeilinger, "Dense coding in experimental quantum communication,” Phys. Rev. Lett. 76, 4656 (1996).

${ }^{11}$ R. Short and L. Mandel, "Observation of sub-Poissonian photon statistics," Phys. Rev. Lett. 51, 384 (1983).

${ }^{12}$ P. Tapster, J. Rarity, and J. Satchell, "Use of parametric down-conversion to generate sub-Poissonian light,” Phys. Rev. A 37, 2963 (1988).

${ }^{13}$ J. Laurat, T. Coudreau, N. Treps, A. Maître, and C. Fabre, "Conditional preparation of a quantum state in the continuous variable regime: Generation of a sub-Poissonian state from twin beams," Phys. Rev. Lett. 91, 213601 (2003).

${ }^{14}$ J. Peřina, O. Haderka, and V. Michálek, "Sub-Poissonian-light generation by postselection from twin beams," Opt. Express 21, 19387-19394 (2013).

${ }^{15}$ S. Singh, "Antibunching, sub-Poissonian photon statistics and finite bandwidth effects in resonance fluorescence," Opt. Commun. 44, 254-258 (1983).

${ }^{16} \mathrm{X}$. Zou and L. Mandel, "Photon-antibunching and sub-Poissonian photon statistics," Phys. Rev. A 41, 475 (1990).

${ }^{17}$ P. Grangier, G. Roger, and A. Aspect, "Experimental evidence for a photon anticorrelation effect on a beam splitter: A new light on single-photon interferences," Europhys. Lett. 1, 173 (1986).

${ }^{18}$ A. B. U'Ren, C. Silberhorn, J. L. Ball, K. Banaszek, and I. A. Walmsley, "Characterization of the nonclassical nature of conditionally prepared single photons," Phys. Rev. A 72, 021802 (2005). 
${ }^{19}$ M. Mirhosseini, O. S. Magaña-Loaiza, M. N. O'Sullivan, B. Rodenburg, M. Malik, M. P. Lavery, M. J. Padgett, D. J. Gauthier, and R. W. Boyd, "Highdimensional quantum cryptography with twisted light," New J. Phys. 17, 033033 (2015).

${ }^{20}$ J. T. Barreiro, T.-C. Wei, and P. G. Kwiat, "Beating the channel capacity limit for linear photonic superdense coding," Nat. Phys. 4, 282 (2008).

${ }^{21}$ A. Kumar, J. Banerji, and R. Singh, "Intensity correlation properties of highorder optical vortices passing through a rotating ground-glass plate," Opt. Lett. 35, 3841-3843 (2010).

${ }^{22}$ A. Kumar, J. Banerji, and R. Singh, "Hanbury Brown-Twiss-type experiments with optical vortices and observation of modulated intensity correlation on scattering from rotating ground glass,” Phys. Rev. A 86, 013825 (2012).

${ }^{23}$ N. Lal, A. Banerji, A. Biswas, A. Anwar, and R. P. Singh, "Single photon sources with different spatial modes," e-print arXiv:1905.01089 [quant-ph] (2019).

${ }^{24}$ C. Gerry, P. Knight, and P. L. Knight, Introductory Quantum Optics (Cambridge University Press, 2005).

${ }^{25}$ R. J. Glauber, "Coherent and incoherent states of the radiation field," Phys. Rev, 131, 2766 (1963).

${ }^{26}$ L. Mandel and E. Wolf, Optical Coherence and Quantum Optics (Cambridge University Press, 1995).

${ }^{27}$ L. Mandel, "Sub-Poissonian photon statistics in resonance fluorescence," Opt. Lett. 4, 205-207 (1979).

${ }^{28}$ M. Lamperti, A. Allevi, M. Bondani, R. Machulka, V. Michálek, O. Haderka, and J. Peřina, "Optimal sub-Poissonian light generation from twin beams by photonnumber resolving detectors," J. Opt. Soc. Am. B 31, 20-25 (2014).

${ }^{29}$ N. Lal, B. Shajilal, A. Anwar, C. Perumangatt, and R. Singh, "Observing sub-Poissonian statistics of heralded single photons using an oscilloscope," in International Conference on Fibre Optics and Photonics (Optical Society of America, 2016), p. Th3A.72

${ }^{30}$ B. I. Cantor and M. C. Teich, "Dead-time-corrected photocounting distributions for laser radiation,” J. Opt. Soc. Am. 65, 786-791 (1975).

${ }^{31}$ E. Jakeman and J. H. Jefferson, "Antibunching and sub-Poissonian statistics in photoelectron-triggered optical dead-time experiments," Opt. Acta 33, 557-576 (1986).

${ }^{32}$ M. Razavi, I. Söllner, E. Bocquillon, C. Couteau, R. Laflamme, and G. Weihs, "Characterizing heralded single-photon sources with imperfect measurement devices,” J. Phys. B: At., Mol. Opt. Phys. 42, 114013 (2009).

${ }^{33}$ B. Blauensteiner, I. Herbauts, S. Bettelli, A. Poppe, and H. Hübel, "Photon bunching in parametric down-conversion with continuous-wave excitation," Phys. Rev. A 79, 063846 (2009).

${ }^{34}$ The source code is available via https://github.com/njlal/Photon_stat-Oscillo scope (MATLAB).

${ }^{35}$ A. Mair, A. Vaziri, G. Weihs, and A. Zeilinger, "Entanglement of the orbital angular momentum states of photons," Nature 412, 313 (2001).

${ }^{36}$ X. Guo, C.-1. Zou, C. Schuck, H. Jung, R. Cheng, and H. X. Tang, "Parametric down-conversion photon-pair source on a nanophotonic chip," Light Sci. Appl. 6, e16249 (2017).

${ }^{37}$ M. Avenhaus, H. B. Coldenstrodt-Ronge, K. Laiho, W. Mauerer, I. A. Walmsley, and C. Silberhorn, "Photon number statistics of multimode parametric down-conversion,” Phys. Rev. Lett. 101, 053601 (2008).

${ }^{38}$ A. M. Yao, "Angular momentum decomposition of entangled photons with an arbitrary pump," New J. Phys. 13, 053048 (2011).

${ }^{39}$ A. Banerji, A. Anwar, H. Sable, N. Lal, and R. P. Singh, "Engineering of orbital angular momentum spectrum of down-converted photons with mode-invariant pump," e-print arXiv:1905.02554 [quant-ph] (2019). 